\title{
Fractional describing function of systems with Coulomb friction
}

\author{
Fernando B. Duarte $•$ J. Tenreiro Machado
}

\begin{abstract}
This paper studies the describing function (DF) of systems constituted by a mass subjected to nonlinear friction. The friction force is decomposed into two components, namely, the viscous and the Coulomb friction. The system dynamics is analyzed in the DF perspective revealing a fractional-order behavior. The reliability of the DF method is evaluated through the signal harmonic contents.
\end{abstract}

Keywords Describing function · Friction · Control · Modeling

\section{Introduction}

The phenomenon of vibration due to friction is verified in many branches of technology where it plays a very useful role. On the other hand, its occurrence is often undesirable, because it causes additional dynamic loads, as well as faulty operation of machines and devices. Despite many investigations that have been car- ried out so far, this phenomenon is not yet fully understood, mainly due to the considerable randomness and diversity of reasons underlying the energy dissipation involving the dynamic effects $[7,8,11,12]$. In this paper, we investigate the dynamics of systems that contain nonlinear friction, namely, the Coulomb forces, in addition to the linear viscous component. Bearing this in mind, the article is organized as follows. Section 2 introduces the fundamental aspects of the describing function method. Section 3 studies the describing function of mechanical systems with nonlinear friction. Finally, Sect. 4 draws the main conclusions and addresses perspectives towards future developments.

\section{Fundamental concepts}

Let us consider the feedback system of Fig. 1, with one nonlinear element $N$ and a linear system with transfer function $G(s)$, where $c(t)$ is the output.

Suppose that the input to a nonlinear element is sinusoidal

$x(t)=X \cos (\omega t)$.

In general, the output of the nonlinear element $y(t)$ is not sinusoidal; nevertheless, the signal $y(t)$ is periodic, with the same period as the input, and containing higher harmonics in addition to the fundamental harmonic component. 


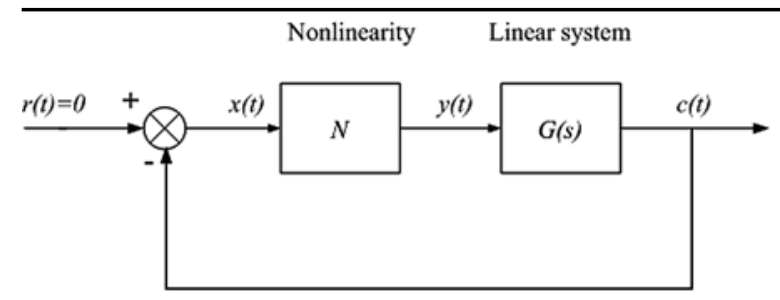

Fig. 1 Nonlinear control system

If we assume that the nonlinearity is symmetrical with respect to the variation around zero, the Fourier series become:

$$
y(t)=\sum_{k=1}^{\infty} Y_{k} \cos \left(k \omega t+\phi_{k}\right)
$$

where $Y_{k}$ and $\varphi_{k}$ are the amplitude and the phase shift of the $k$ th harmonic component of the output $y(t)$, respectively.

In the DF analysis, we assume that only the fundamental harmonic component of the output is significant. Such assumption is often valid since the higher harmonics in the output of a nonlinear element are usually of smaller amplitude than the fundamental component $[4,13,16]$. Moreover, most systems are "lowpass filters" with the result that the higher harmonics are further attenuated $[1,3,6]$.

The DF, or sinusoidal DF, of a nonlinear element, $N(X, \omega)$, is defined as the complex ratio of the fundamental harmonic component of the output and the input, that is:

$$
N(X, \omega)=\frac{Y_{1}}{X} e^{j \phi 1}
$$

where the symbol $N$ represents the DF, $X$ is the amplitude of the input sinusoid, and $Y_{1}$ and $\varphi_{1}$ are the amplitude and the phase shift of the fundamental harmonic component of the output, respectively. Several analytical expressions of DF of standard nonlinear elements can be found in the references $[2,5,9]$.

For nonlinear systems without involving energy storage the DF is merely amplitude-dependent, that is $N=N(X)$. However, when we have nonlinear elements that involve energy, the DF method is both amplitude and frequency dependent yielding $N=$ $N(X, \omega)$. In this case, to determine the DF, usually we have to adopt a numerical approach because it is impossible to find a closed-form analytical solution. Once calculated, the DF can be used for the approximate stability analysis of the nonlinear control system.

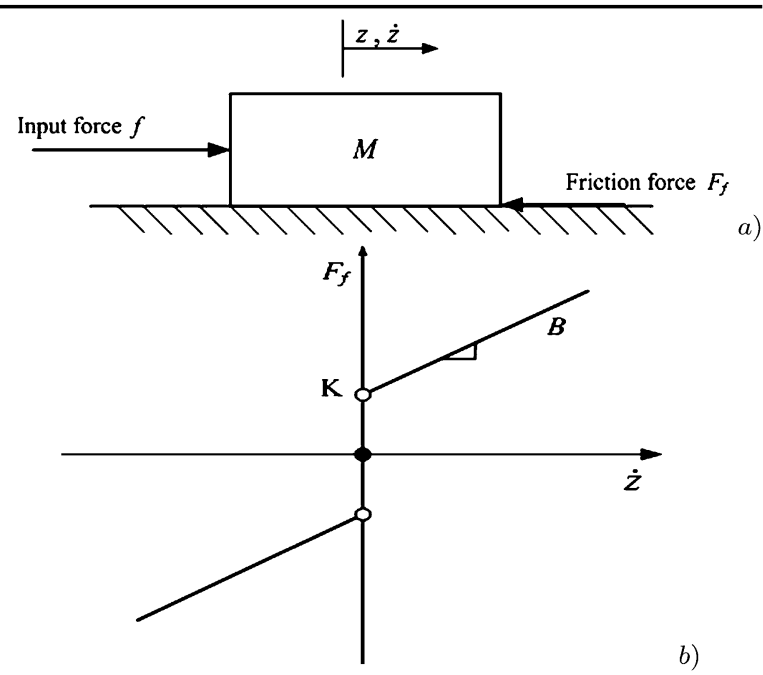

Fig. 2 (a) Elemental mass system subjected to nonlinear friction, and (b) non-linear friction with Coulomb and viscous components (CV model)

Let us consider again the standard control system shown in Fig. 1 where the block $N$ denotes the DF of the nonlinear element. If the higher harmonics are sufficiently attenuated, $N$ can be treated as a real or complex variable gain and the closed-loop frequency response becomes

$$
\frac{C(j \omega)}{R(j \omega)}=\frac{N(X, \omega) G(j \omega)}{1+N(X, \omega) G(j \omega)} .
$$

The characteristic equation is

$$
1+N(X, \omega) G(j \omega)=0 .
$$

If (5) can be satisfied for some values of $X$ and $\omega$, then a limit cycle is predicted for the nonlinear system. Moreover, since (5) is valid only if the nonlinear system is in a steady-state limit cycle, the DF analysis predicts only the presence or the absence of a limit cycle and cannot be applied to the analysis of other types of time responses.

\section{Mechanical systems with nonlinear friction}

In this section, we analyze the DF of a dynamical system with nonlinear friction composed of a combination of the viscous and Coulomb components.

Let us consider a system (Fig. 2) with a mass $M$, moving on a horizontal plane under the action of an 
Fig. 3 The Nichols plot of $N(F, \omega)$ for the system subjected to nonlinear friction (CV model) with $M=1.0 \mathrm{~kg}$, $2.5 \leq F \leq 100.0 \mathrm{~N}$, $1.0 \leq \omega \leq 100.0 \mathrm{rad} \mathrm{s}^{-1}$ with

$\{B, K\}=\left\{0.5 \mathrm{~N} \mathrm{~s} \mathrm{~m}^{-1}, 2.0 \mathrm{~N}\right\}$

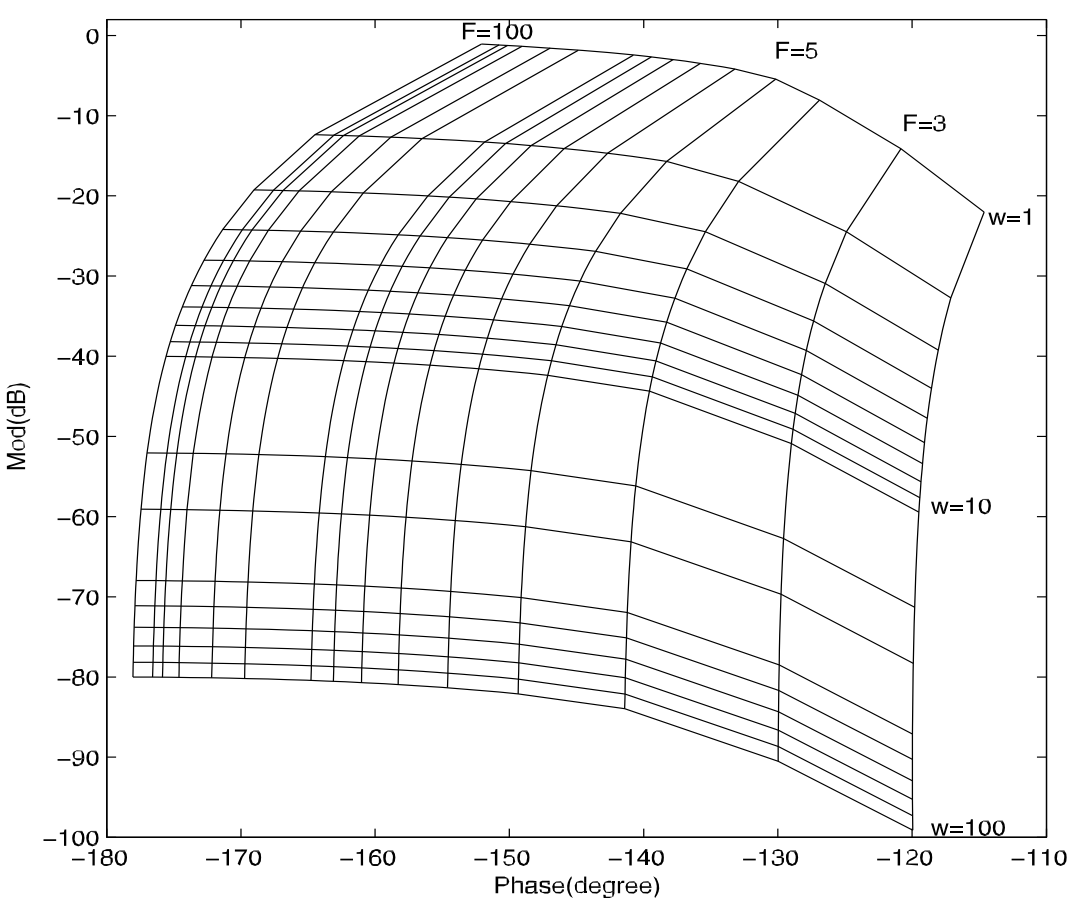

input force $\boldsymbol{f}(t)$, with a friction $F_{\boldsymbol{f}}(t)$ effect composed of two components: a non-linear Coulomb $K$ part and a linear viscous $B \dot{z}$ part (so-called $\mathrm{CV}$ model).

The equation of motion in this system is as follows:

$$
M \ddot{z}(t)+F_{f}(t)=f(t)
$$

where $z, \dot{z}$ and $\ddot{z}$ are the displacement, velocity and acceleration, respectively.

For the system of Fig. 2 we can consider as input a sinusoidal force $f(t)$ applied to mass $M$ and as output the position $z(t)$. Therefore, the describing function $N(F, \omega)$ can be calculate numerically as:

$$
\begin{aligned}
& f(t)=F \cos (\omega t), \\
& z(t)=\sum_{k=1}^{\infty} Z_{k} \cos \left(k \omega t+\phi_{k}\right), \\
& N(F, \omega)=\frac{Z_{1}}{F} e^{j \phi_{1}} .
\end{aligned}
$$

Figure 3 shows the Nichols plot of $N(F, \omega)$ for $M=1.0 \mathrm{~kg}, B=0.5 \mathrm{~N} \mathrm{~s} \mathrm{~m}^{-1}$ and $K=2.0 \mathrm{~N}$. Alternatively Figs. 4 and 5 illustrate the $\log -\log$ plots of $|\operatorname{Re}\{N\}|$ and $|\operatorname{Im}\{N\}|$ vs. the exciting frequency $\omega$, for different values of the input force $2.5 \leq F \leq$
100.0 N. We have different results according to the excitation force $F$ and we get straight lines with slopes revealing clearly a fractional-order behavior.

Figure 6 depicts the harmonic content of the output signal $z(t)$ for an input force of $F=10 \mathrm{~N}$. We verify that the output signal has a half-wave symmetry because the harmonics of an even order are negligible. Moreover, the fundamental component of the output signal is the most important one, while the amplitude of the high order harmonics decay significantly. Therefore, we can conclude that, for the friction $\mathrm{CV}$ model, the DF method may lead to a good approximation.

In order to study $\operatorname{Re}\{N(F, \omega)\}$ and $\operatorname{Im}\{N(F, \omega)\}$, we approximate the numerical results through power functions:

$$
\begin{array}{ll}
\operatorname{Re}\{N(F, \omega)\}=-a \omega^{-b}, & \{a, b\} \in \mathbb{R}^{+}, \\
\operatorname{Im}\{N(F, \omega)\}=-c \omega^{-d}, & \{c, d\} \in \mathbb{R}^{+} .
\end{array}
$$

Figure 7 illustrates the variation of the parameters $\{a, b\}$ and $\{c, d\}$ versus $F$ for $K=\{1.0,2.0,3.0,4.0$, 5.0\}. We verify that $\operatorname{Re}\{N(F, \omega)\}$ and $\operatorname{Im}\{N(F, \omega)\}$ reveal a distinct relationship with $\omega$ [10]. In fact, we conclude that $\operatorname{Re}\{N\}$ and $\operatorname{Im}\{N\}$ are, in the two cases, 
Fig. 4 Log- $\log$ plots of $|\operatorname{Re}\{N\}|$ vs the exciting frequency $1.0 \leq \omega \leq 100$ $\operatorname{rad~s}^{-1}$, for the CV model with

$\{B, K\}=\left\{0.5 \mathrm{~N} \mathrm{~s} m^{-1}, 2.0 \mathrm{~N}\right\}$,

$M=1.0 \mathrm{~kg}$ and

$F=\{5,15,30,100\} \mathrm{N}$

Fig. 5 Log- $\log$ plots of $|\operatorname{Im}\{N\}| v s$ the exciting frequency $1.0 \leq \omega \leq 100$ $\operatorname{rad~s}^{-1}$, for the $\mathrm{CV}$ model with

$\{B, K\}=\left\{0.5 \mathrm{~N} \mathrm{~s} \mathrm{~m}^{-1}, 2.0 \mathrm{~N}\right\}$,

$M=1.0 \mathrm{~kg}$ and

$F=\{5,15,30,100\} \mathrm{N}$
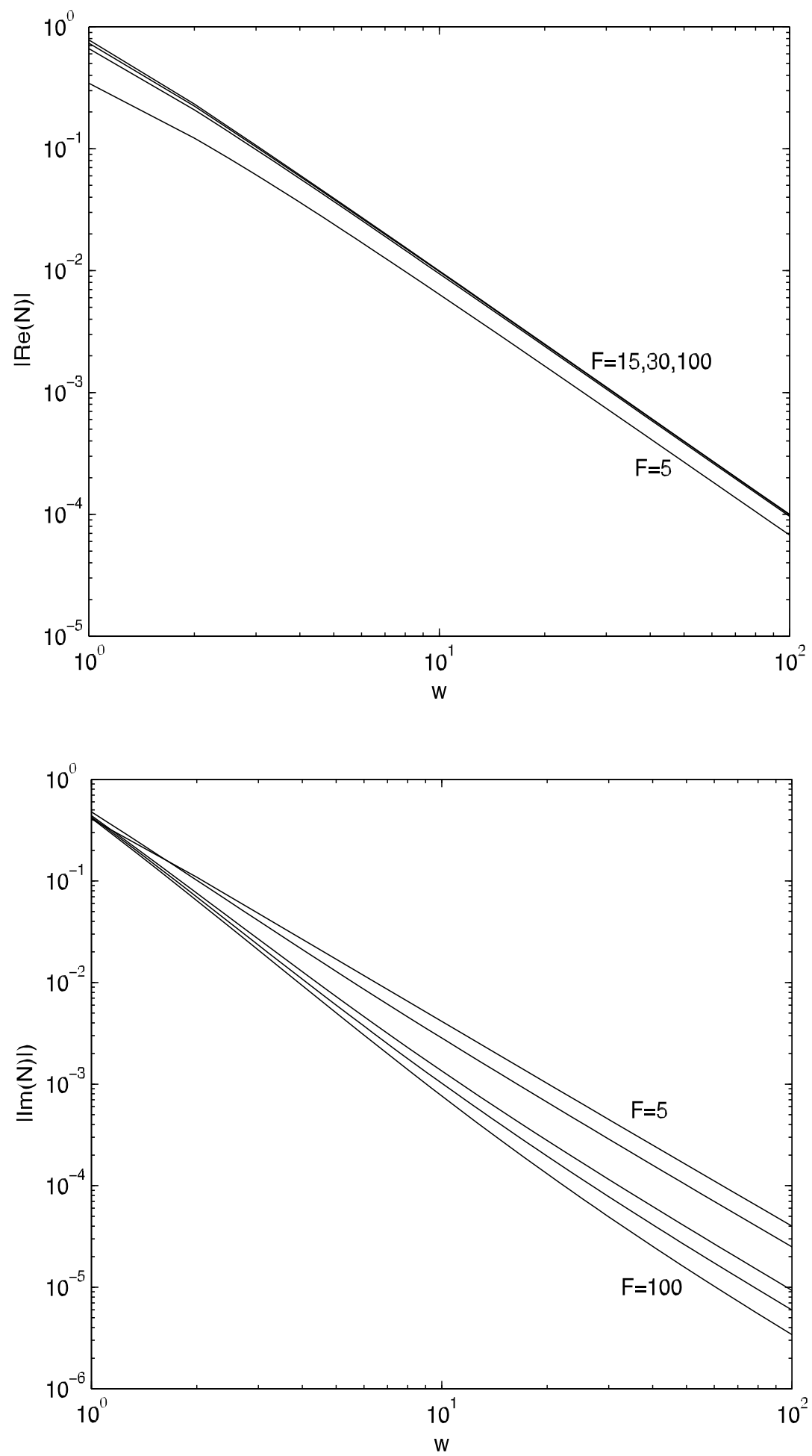

of the same type, following the power law according to expression (8). Furthermore, we obtain fractional- order dynamics as revealed by the Nichols chart in Fig. 3. Nevertheless, $\operatorname{Re}\{N(F, \omega)\}$ has an integer na- 
Fig. 6 The Fourier transform of the output position $z(t)$, for the $\mathrm{CV}$ model, $v s$. the exciting frequency $1.0 \leq \omega \leq 100.0$ $\operatorname{rad~s}^{-1}$ and the harmonic frequency index

$k=\{1,3,5,7,9\}$ for an input force $F=20 \mathrm{~N}$, with $M=1.0 \mathrm{~kg}$, $\{B, K\}=\left\{0.5 \mathrm{~N} \mathrm{~s} \mathrm{~m}^{-1}, 2.0 \mathrm{~N}\right\}$

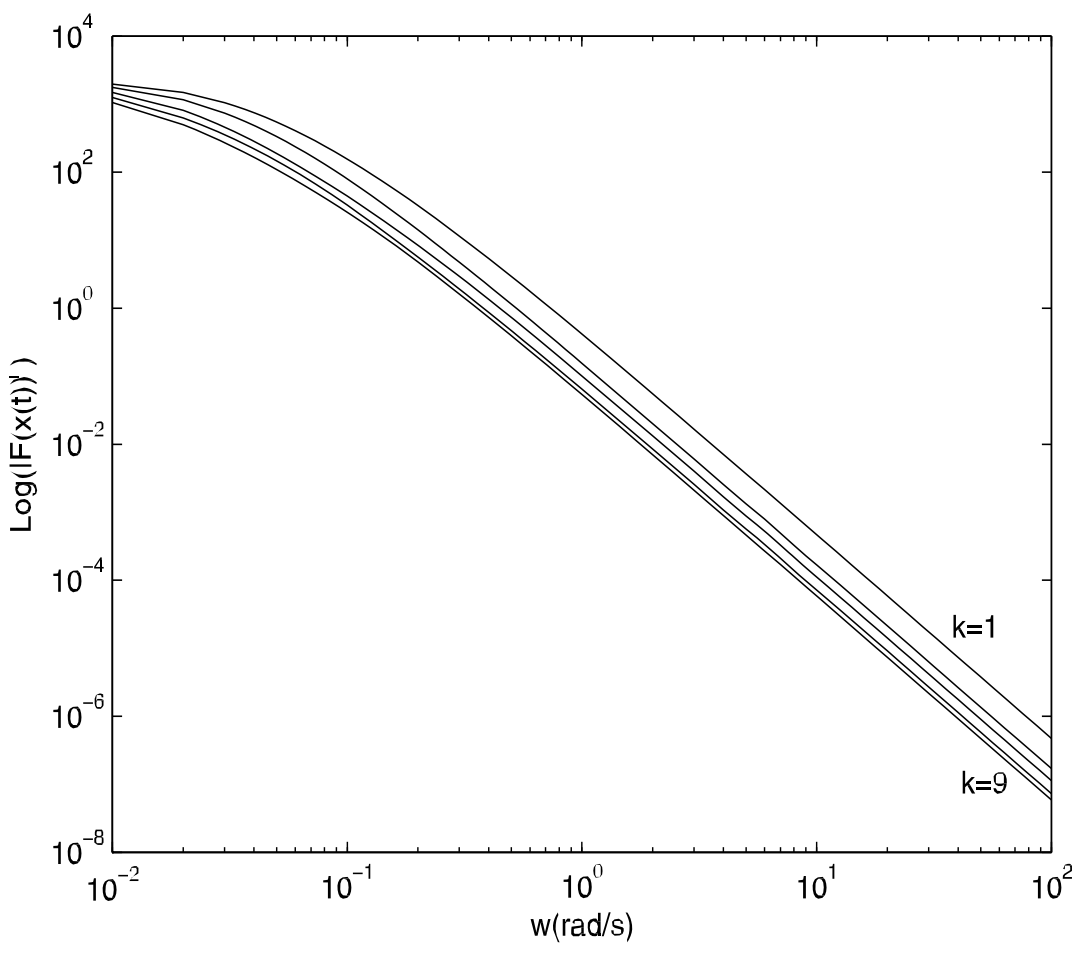

ture with $b \approx 2$, while $\operatorname{Im}\{N(F, \omega)\}$ is clearly fractional with $2<d<2.7[14,15]$.

To have a deeper insight into the effects of the different CV components, several complementary experiments were performed by separately varying the values of $K$ and $M$ while keeping the rest of components constant. For example, Fig. 8 presents the values of the parameters $\{a, b, c, d\}$ when approximating $\operatorname{Re}\{N\}$ and $\operatorname{Im}\{N\}$, for $2.5 \leq F \leq 100.0 \mathrm{~N}$ with $M=\{0.5$, $1.0,2.0,3.0\} \mathrm{kg}$ and $\{B, K\}=\left\{0.5 \mathrm{~N} \mathrm{~s} \mathrm{~m}^{-1}, 2.0 \mathrm{~N}\right\}$.

As we should expect, $\operatorname{Re}\{N(F, \omega)\}$ and $\operatorname{Im}\{N(F, \omega)\}$ vary with the system parameters, but we conclude that the integer $v s$ fractional behaviors remain identical, respectively. Furthermore, the fractional characteristics of $\operatorname{Im}\{N\}$ are a direct consequence of the nonlinear action of the Coulomb friction, since the viscous friction leads simply to a of a linear integer order.

\section{Conclusions}

This paper addressed the study of systems with nonlinear friction. The dynamics of elemental mechanical system was analyzed by describing the function method and comparing with standard models. The polar plot reveals a fractional-order behavior which was further analyzed in the real and imaginary components. The results encourage a further study of nonlinear systems in a similar perspective and the adoption of the tools of fractional calculus. The fractional-order behavior is clearly a result of the Coulomb friction. In fact, for a linear system the DF yields the transfer function, which for the mass and linear friction system is simply

$$
N(\omega)=\frac{\mathcal{F}\{z(t)\}}{\mathcal{F}\{f(t)\}}=\frac{1}{-M \omega^{2}+j B \omega}
$$

where $F$ is the Fourier operator. The asymptotic cases $\omega \rightarrow 0 \Rightarrow N(\omega) \rightarrow-j B^{-1}$ and $\omega \rightarrow \infty \Rightarrow N(\omega) \rightarrow$ $-M^{-1} \omega^{-2}$ lead, clearly, to integer-order dynamics. However, the introduction of the Coulomb component with amplitude $K$, involves signal excursions around the zero value that can be interpreted as an averaging of the friction effect. The signal excursion, and consequently the friction averaging, depends on the frequency and changes smoothly with it. This smooth change results in a fractional dynamics that cannot be attended by the classical analysis. 

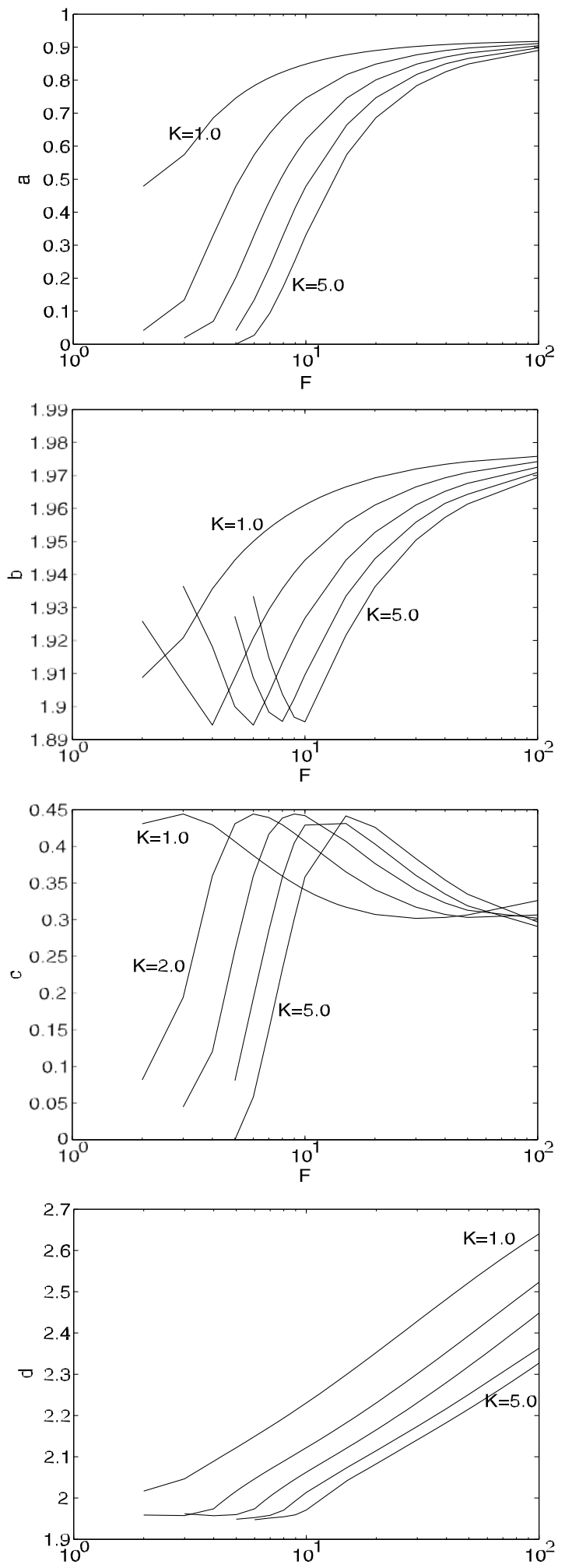

Fig. 7 Variation of the parameters $\{a, b\}$ and $\{c, d\}$ vs $2.5 \leq F \leq 100.0 \mathrm{~N}$, in the $\mathrm{CV}$ model with $M=1.0 \mathrm{~kg}$, $B=0.5 \mathrm{~N} \mathrm{sm}^{-1}$ and $K=\{1.0,2.0,3.0,4.0,5.0\} \mathrm{N}$
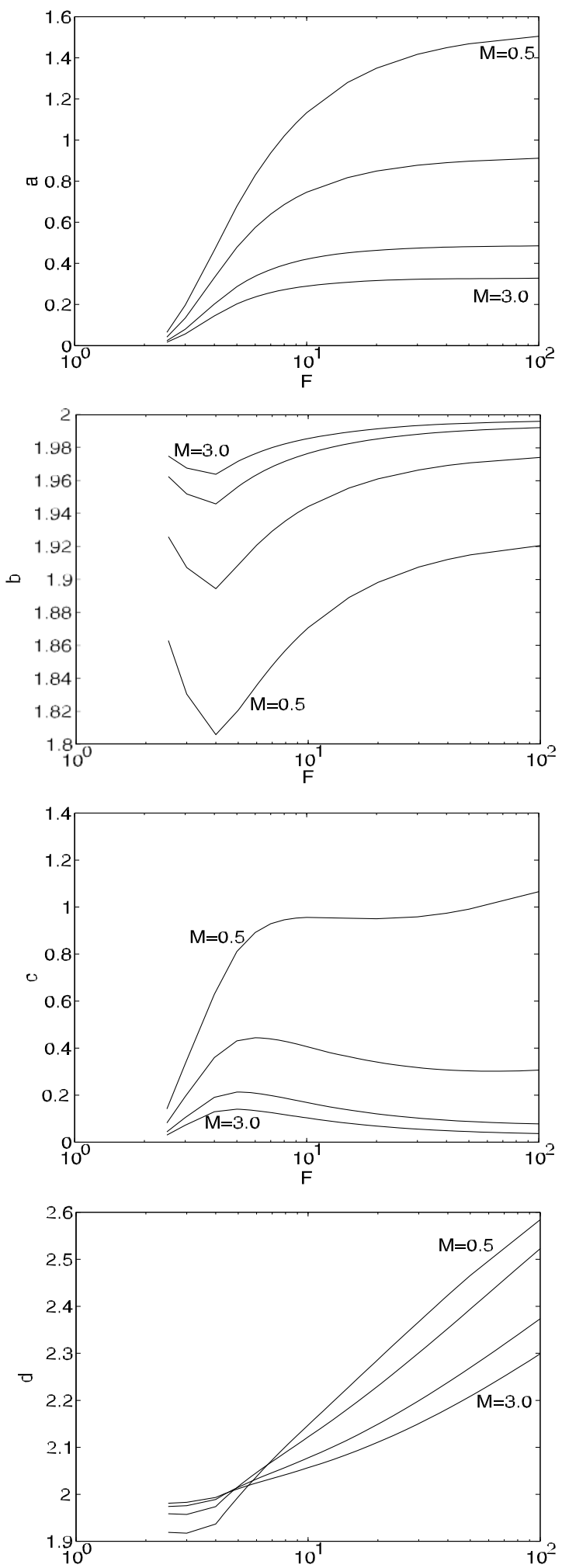

Fig. 8 Variation of the parameters $\{a, b\}$ and $\{c, d\}$ vs $2.5 \leq F \leq 100.0 \mathrm{~N}$, in the CV model with $M=\{0.5,1.0,2.0$, $3.0\} \mathrm{kg},\{B, K\}=\left\{0.5 \mathrm{~N} \mathrm{~s} \mathrm{~m}^{-1}, 2.0 \mathrm{~N}\right\}$ 
Acknowledgements The authors thank GECAD-Grupo de Investigação em Engenharia do Conhecimento e Apoio à Decisão, for the support to this work.

\section{References}

1. Atherton, D.P.: Nonlinear control engineering. In: IEEE, 1st International Conference on Electrical Engineering. Van Nostrand Reinhold Company, London (1975)

2. Karnopp, D.: Computer simulation of stick-slip friction in mechanical dynamic systems. ASME J. Dyn. Syst. Meas. Control 107, 100-103 (1985)

3. Cox, C.S.: Algorithms for limit cycle prediction: a tutorial paper. Int. J. Electr. Eng. Education 24, 165-182 (1987)

4. Slotine, J.E., Li, W.: Applied Nonlinear Control. PrenticeHall, New Jersey (1991)

5. Haessig, D.A., Friedland, B.: On the modelling and simulation of friction. ASME J. Dyn. Syst. Meas. Control 113, 354-362 (1991)

6. Dupont, P.E.: The effect of Coulomb friction on the existence and uniqueness of the forward dynamics problem, pp. 1442-1447 (1992)

7. Armstrong, B., Dupont, B., de Wit, C.: A survey of models, analysis tools and compensation methods for the machines with friction. Automatica 30, 1083-1183 (1994)

8. Armstrong, B., Amin, B.: Pid control in the presence of static friction: a comparison of algebraic and describing function analysis. Automatica 32, 679-692 (1996)
9. Azenha, A., Machado, J.A.: On the describing function method and prediction of limit cycles in nonlinear dynamical systems. Syst. Anal. Model. Simul. 33, 307-320 (1998)

10. Podlubny, I.: Fractional Differential Equations. Academic Press, San Diego (1999)

11. Barbosa, R., Machado, J.A.: Describing function analysis of systems with impacts and backlash. Nonlinear Dyn. 29, 235-250 (2002)

12. Barbosa, R., Machado, J.A., Ferreira, I.: Describing function analysis of mechanical systems with nonlinear friction and backlash phenomena. In: 2nd IFAC Workshop on Lagrangian and Hamiltonian Methods for Non-Linear Control. Sevilla, Spain (2003)

13. Lanusse, P., Oustaloup, A.: Wind-up compensation system for fractional controller. In: First IFAC Workshop on Fractional Differentiation and its Applications. Bordeaux, France (2004)

14. Duarte, F., Machado, J.A.: Describing function method in nonlinear friction. In: IEEE, 1st International Conference on Electrical Engineering. Coimbra, Portugal (2005)

15. Duarte, F., Machado, J.A.: Fractional dynamics in the describing function analysis of nonlinear friction. In: 2nd IFAC Workshop on Fractional Differentiation and Its Applications. Porto, Portugal (2006)

16. Vinagre, B.M., Monge, C.A.: Reset and fractional integrators in control applications. In: 8th International Carpathian Control Conference. High Tatras, Slovak Republic (2007) 